\title{
Continuous Line Drawings via the Traveling Salesman Problem
}

\author{
Robert Bosch and Adrianne Herman *
}

August 13, 2003

\begin{abstract}
We describe how to use the traveling salesman problem (TSP) to create continuous line drawings of target pictures.
\end{abstract}

\section{Introduction}

If you ever took a drawing class, even as a child, chances are good that at some point you made a continuous line drawing. (Art teachers are fond of them.) When you did this, you looked at the object you were asked to draw, you placed the tip of your drawing implement on your paper, and you made a line drawing of the object, taking great care to keep the tip of your drawing implement in contact with your paper until you were finished.

When you were making your continuous line drawing, you were solving an optimization problem. Your objective was to produce the best possible line drawing of the object. What made this difficult was the constraint: you were not allowed to remove your drawing implement from the paper until your drawing was complete.

In this brief note we describe how to construct TSP instances whose solution yield continuous line drawings of target pictures.

\section{The Procedure}

To create a continuous line drawing of a target picture, we do the following:

Step 1: Format the target picture. Resize the target picture so that it is $k m \times k n$ (i.e., so that it has $k m$ rows and $k n$ columns of pixels). Then convert it into PGM (portable graymap) format. In PGM format, each pixel has a grayscale value between 0 (completely black) and 255 (completely white).

Step 2: Divide the target picture into squares. Partition the pixels of the target picture into $m$ rows and $n$ columns of $k \times k$ squares. For each row $i$

\footnotetext{
${ }^{*}$ Dept. of Mathematics, Oberlin College, Oberlin, Ohio, 44074 (bobb@cs.oberlin.edu).
} 
and column $j$, compute the mean grayscale value $\mu_{i j}$ of the pixels in square $(i, j)$. Then set $g_{i j}=\gamma-\left\lfloor\gamma \mu_{i j} / 256\right\rfloor$. Note that $g_{i j}$ is the average darkness of square $(i, j)$ on a 0 (completely white) to $\gamma$ (completely black) grayscale.

Step 3: Construct and solve the TSP instance. Divide the canvas into $m$ rows and $n$ columns of squares. For each row $i$ and column $j$, randomly place $g_{i j}$ cities (points) in square $(i, j)$. Do this in such a way that the cities in square $(i, j)$ are uniformly distributed in square $(i, j)$. After computing the intercity distances, solve the TSP instance and plot the tour.

Figure 1 displays a continuous line drawing produced via this TSP-based procedure. The target picture was a $200 \times 200$ picture of Marilyn Monroe. We used $m=n=100, k=2$, and $\gamma=4$. We used John Bradley's XV package [2] to resize and format the target picture and the chained Lin-Kernighan heuristic in Applegate, Bixby, Chvátal, and Cook's Concorde [1] package to "solve" the TSP instance. (We have no proof that our tour is optimal.)

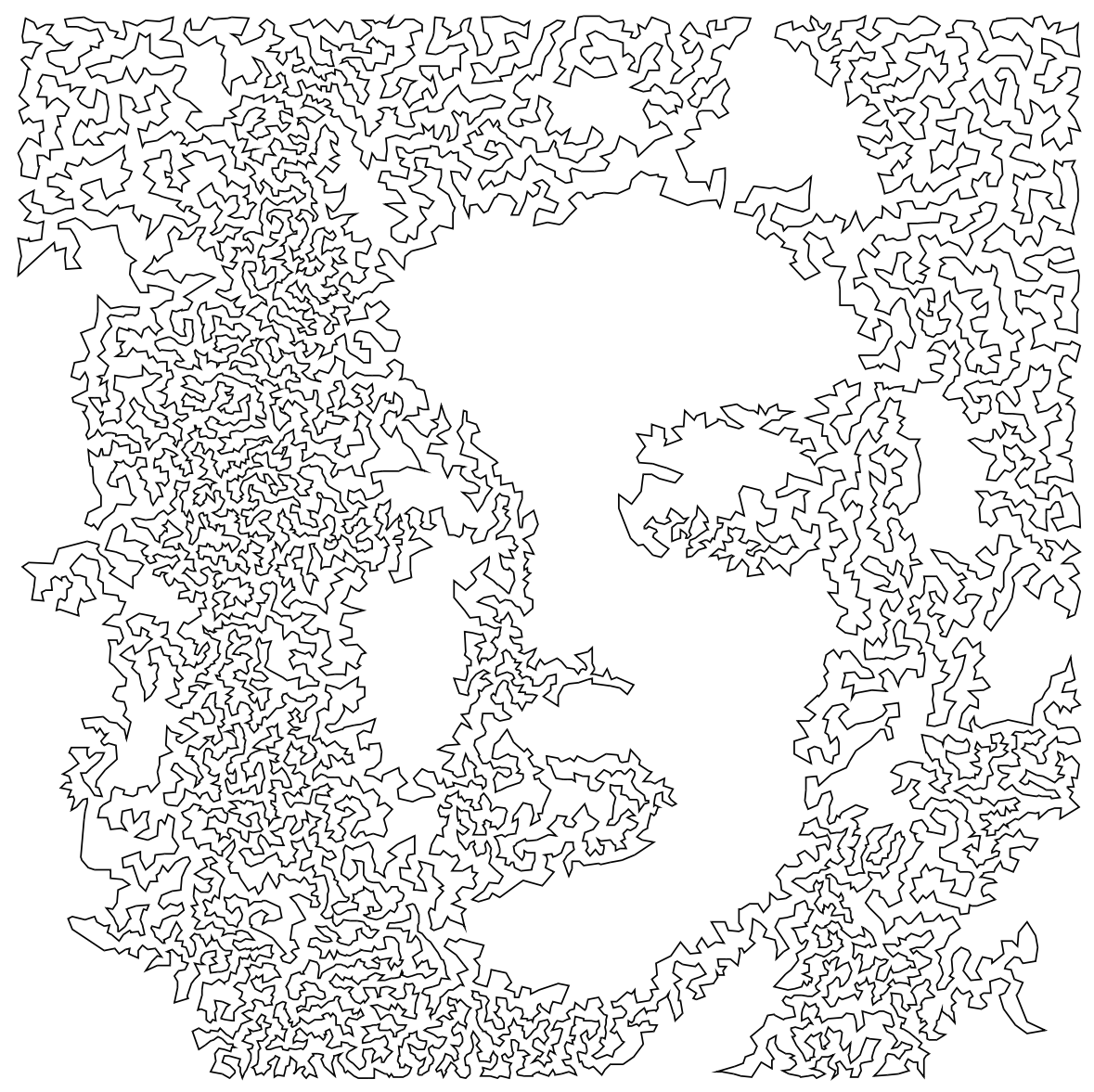

Figure 1: Marilyn Monroe, 11508 cities

\section{References}

[1] D. Applegate, R. Bixby, V. Chvátal, and W. Cook, Concorde - a code for solving Traveling Salesman Problems, http://www.princeton.edu/tsp/concorde.html.

[2] J. Bradley, The On-Line Version of the XV 3.10a Manual, http://www.icgeb.trieste.it/ netsrv/xvman/index.html. 